\title{
Decoupling Control Design for the Module Suspension Control System in Maglev Train
}

\author{
Guang He, Jie Li, and Peng Cui \\ College of Mechatronics Engineering and Automation, National University of Defense Technology, Changsha 410073, China \\ Correspondence should be addressed to Jie Li; lj@maglev.cn
}

Received 26 August 2014; Revised 11 December 2014; Accepted 26 December 2014

Academic Editor: Dan Ye

Copyright (C) 2015 Guang He et al. This is an open access article distributed under the Creative Commons Attribution License, which permits unrestricted use, distribution, and reproduction in any medium, provided the original work is properly cited.

\begin{abstract}
An engineering oriented decoupling control method for the module suspension system is proposed to solve the coupling issues of the two levitation units of the module in magnetic levitation (maglev) train. According to the format of the system transfer matrix, a modified adjoint transfer matrix based decoupler is designed. Then, a compensated controller is obtained in the light of a desired close loop system performance. Optimization between the performance index and robustness index is also carried out to determine the controller parameters. However, due to the high orders and complexity of the obtained resultant controller, model reduction method is adopted to get a simplified controller with PID structure. Considering the modeling errors of the module suspension system as the uncertainties, experiments have been performed to obtain the weighting function of the system uncertainties. By using this, the robust stability of the decoupled module suspension control system is checked. Finally, the effectiveness of the proposed decoupling design method is validated by simulations and physical experiments. The results illustrate that the presented decoupling design can result in a satisfactory decoupling and better dynamic performance, especially promoting the reliability of the suspension control system in practical engineering application.
\end{abstract}

\section{Introduction}

As an urban track transportation vehicle with large application prospect, low speed maglev trains have been developed for almost 30 years $[1,2]$ and there have already been several commercial operation lines or test lines [38]. Maglev train utilizes suspension controllers to adjust the electromagnetic forces between the electromagnets and the track for stable levitation. Hence, the electromagnetic suspension control system is the most pivotal component of the maglev train, which attracts tremendous attention [9-12]. As of today, the stability problem of the suspension control system has been basically solved. The major work on suspension control is excepted be transferred to the performance promotion and the practical problem existing in engineering applications. On the basis of the mechanical decoupling in bogie, levitation modules can be considered as the foundational elements of the low speed maglev train. At present, the main existing suspension control methods decompose the module into two single-suspension-control units. However, due to the physical stiffness structure of levitation module, direct coupling between the two singlesuspension-control units will attenuate disturbance rejection capability of the levitation control system and also becomes a serious obstacle to the performance promotion. To some extent, the adjustment of one levitation unit may destabilize the whole module suspension system. Therefore, it is essential to develop some decoupling control strategy for the module suspension control system.

By viewing the module as an integrated object, the suspension control system is a two-input-two-output (TITO) control system. The engineering oriented research on the decoupling control of the module suspension system has rarely been reported. Fortunately, considerable efforts have already been devoted to the decoupling control of the multiple-input-multiple-output (MIMO) system for several decades. Different control strategies have been developed to overcome the complicated couplings between control loops, such as inverse Nyquist array [13], internal model control [14], inverse based decoupling control [15], and other decoupler based methods. Those methods can allow parameter perturbation and uncertainties in system model with robust 
requirement, which benefits the decoupling design of the module suspension system. Besides, although the differential geometry technique is also a feasible approach to deal with the multivariable decoupling control problems [16, 17], their need for precise mathematical model is an obstacle to apply it to practical engineering. The general decoupling control approach is to design the decoupler so that the MIMO control system can be treated as multiple single-input-single-output (SISO) loops, which allows us to use well developed single loop controller design methods. Many decoupling control design methods are developed based on this view as well [1822]. The ideal decoupler is to be designed as the inverse of the transfer function matrix. However, this kind of decoupler needs to calculate the inverse of the process transfer function matrix resulting in too complicated calculation. Shen et al. considered the adjoint transfer matrix of the original multivariable system as the decoupler [23], where it can avoid complicated computation, especially for TITO system.

In this paper, a modified adjoint transfer matrix based decoupler was presented. First, the existing coupling in the module suspension control system is analyzed and the dynamic model is also given. By adopting the modified adjoint transfer matrix as the decoupler, we divide the module suspension control system into two independent SISO control systems. Then, compensated controllers are designed to meet the desired loop performance and robustness demand of the module suspension control system. The formulation of a resultant decoupling controller is obtained by combing the decoupler and the compensated controller. Multivariable PID structure controller is the most effective technology in engineering applications because of adequate performance with simple structure $[18,19,24,25]$, which is also adopted in our practical CMS04 low speed maglev train. Hence, the resultant decoupling controller is transformed to PID type controller by model reduction. Given the parameters uncertainties and nonlinear characteristic in the magnetic suspension system, the modeling errors between the linearized model and practical physical model have been taken into account. The modeling errors are measured by frequency sweeping experiments on a real full-scale single bogie of CMS-04 maglev train, based on which the robust stability of the decoupled module suspension control system is validated. Furthermore, simulations and experimental results show that the proposed decoupling method can be well applied in the module suspension system and promote the suspension capability in practical engineering application.

The rest of the paper is organized as follows. The coupling analysis and dynamic model of the module suspension system are given in Section 2. Section 3 describes the decoupling design procedure in detail. The case study in Section 4 is to determine the parameters of the designed decoupling controller. Simulations and experiments are presented in this section. Finally, Section 5 gives the conclusions of this paper.

\section{Coupling Analysis and Modeling of the Module Suspension System}

The low speed electromagnetic suspension (EMS) vehicle consists of cabin body, levitation bogies, secondary

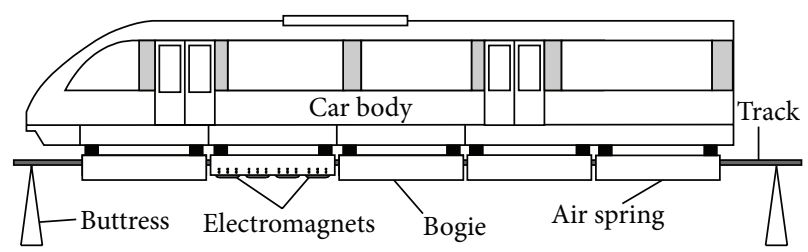

FIgURE 1: Lateral view of the CMS04 low speed maglev vehicle.

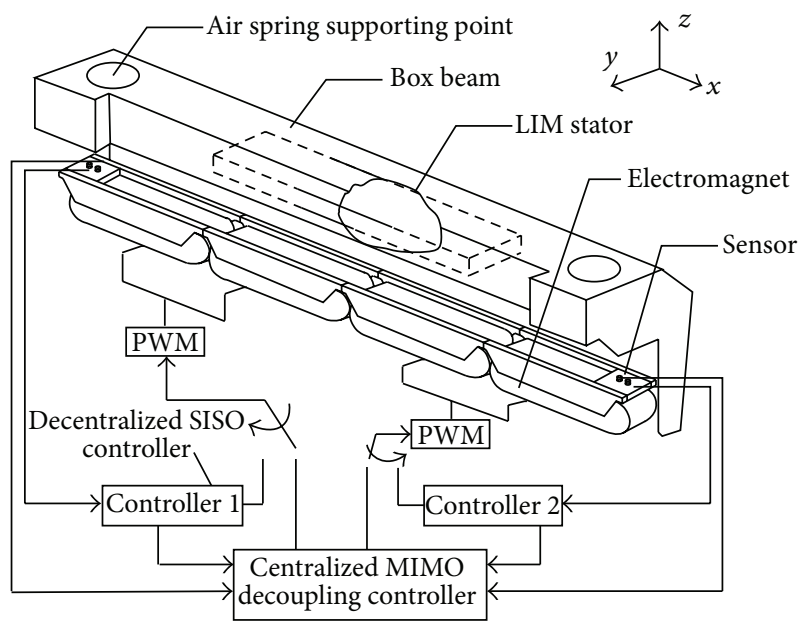

Figure 2: Schematic of the module suspension control system.

suspensions, and levitation and guidance magnets. A lateral view of the CMS04 low speed maglev vehicle is shown in Figure 1 where it can be founded that the car body is supported by five bogies, with each bogie consisting of two levitation modules. Each module contains two pairs of adjacent electromagnets which are controlled by decentralized SISO controllers; thus, there are a total of four levitation units in a bogie.

As the bogie is the pivotal component of the maglev vehicle, analyzing the coupling issue of the bogie is essential to design decoupling methods. The coupling issue of the bogie was investigated through static experiments in the CMS-04 low speed maglev vehicle in [26], from which it can be founded that the coupling between the two levitation units in a module is much stronger ([26], Figure 3). Therefore, the paper focuses on the coupling between the two levitation units in one levitation module. The schematic of the module suspension control system is shown in Figure 2, where it gives two kinds of controller construction: the decentralized SISO controller and the centralized MIMO decoupling controller. The former is the common control methodology in maglev system. It utilizes only the sensors message of one levitation unit to realize its stable levitation, which leads to the fact that adjusting the movement of one levitation control unit affects the performance of the other one because of the module's stiff structure. To cope with this problem, a centralized MIMO decoupling control scheme described in Figure 2 is presented and will be discussed in detail. This kind of controller can use both sensors messages of the levitation units in a module to produce appropriate control laws. 
Generally, in the dynamic modeling of the levitation module, only that the degrees of freedom in pitch and in vertical direction are considered. With the purpose of engineering application, some assumptions can be made below when developing the dynamic model of the levitation module [27].

(1) The mass distribution of the levitation module is homogenous, and the gravity centre of levitation module coincides with its geometrical centre. The track is considered to be stiff, so the flexible distortion of the track can be neglected.

(2) The magnetic leakage and edge effect of the electromagnet are neglected. That is to say, the total magnetic potential during the levitation gap is distributed evenly in the $x$ direction.

(3) The uniformly distributed electromagnet force can be equated with two concentrated forces acting on the centre of levitation units in one levitation module. Besides, the force transferred from air spring applies on the measuring point of the gap sensor in the $y$ direction.

Based on the assumptions above, the force diagram of the levitation module in lateral is given in Figure $3 . F_{i}$ is the electromagnet force of the $i$ th levitation unit; $z_{i}$ is the gap between the track and the module at the electromagnet force acting spot of the levitation unit $i ; N_{i}$ is the force of the $i$ th levitation unit transferred from air spring; $\delta_{i}$ is the measured gap values of the gap sensors; $\theta$ is the pitch angle of the levitation module; $l$ is the length of the electromagnet of one module; $c$ is the gap between the centre of the levitation module and the track; $m$ is the total mass of one levitation module.

The geometrical relationship of the levitation module is given as follows:

$$
\begin{array}{cc}
c=\frac{\left(\delta_{1}+\delta_{2}\right)}{2}, & \theta=\frac{\left(\delta_{1}-\delta_{2}\right)}{l}, \\
z_{1}=\frac{\left(3 \delta_{1}+\delta_{2}\right)}{4}, & z_{2}=\frac{\left(\delta_{1}+3 \delta_{2}\right)}{4} .
\end{array}
$$

The motions of the levitation module contain the rotation around the mass centre $O$ in the $x-z$ plane and the movement in the vertical direction. According to Newton's law, the motion equation of the levitation module is described as follows:

$$
\begin{gathered}
m g-F_{1}-F_{2}+N_{1}+N_{2}=m \ddot{c}, \\
F_{2} \frac{l}{4} \cdot \cos \theta-F_{1} \frac{l}{4} \cdot \cos \theta+N_{1} \frac{l}{2} \cdot \cos \theta-N_{2} \frac{l}{2} \cdot \cos \theta=J \ddot{\theta},
\end{gathered}
$$

where $J$ is the rotary inertia of the levitation module in the pitch direction, $F_{i}=k_{e} I_{i} / z_{i}, k_{e}=\mu_{0} N^{2} A / 2, I_{i}$ is the coil current of the electromagnets of the $i$ th levitation unit, $N$ is the number of turns of electromagnet winding, $A$ is the pole area, $\mu_{0}$ is the permeability of vacuum, and $g$ is the acceleration due to gravity.

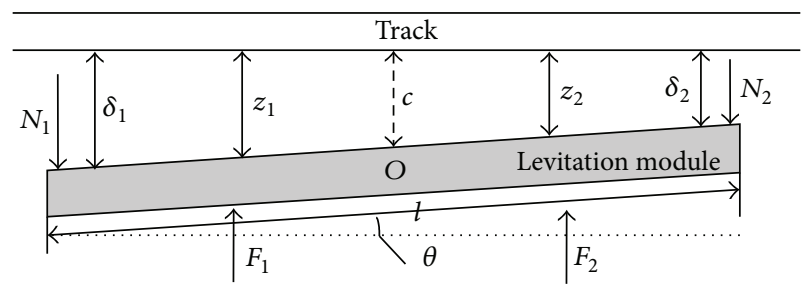

FIgURE 3: The force diagram of one levitation module in lateral.

The relationship between the current and the voltage of the electromagnets is derived as

$$
u_{i}=R I_{i}+\frac{2 k_{e}}{z_{i}} \dot{I}_{i}-\frac{2 k_{e}}{z_{i}^{2}} I_{i} \dot{z}_{i}, \quad i=1,2,
$$

where $u_{i}$ and $R$ are the voltage and direct current (DC) resistance of the electromagnet of the $i$ th levitation unit, respectively.

Equations (1) (3) describe the dynamic behaviour of the levitation module. However, the magnetic levitation system has been pointed to be inherently unstable without active control. In this paper, the decoupling control methods are developed on the basis of that the suspension system is already stable. Hence, an additional controlling force is commonly used to stabilize the single levitation unit. Here, the PD controller is adopted and the feedback control law is given as follows:

$$
I_{e i}=k_{p}\left(\delta_{i}-r_{d}-\delta_{0}\right)+k_{d} \dot{\delta}_{i}, \quad i=1,2,
$$

where $k_{p}$ and $k_{d}$ are the proportional and derivative control coefficients, $I_{e i}$ is the desired current, $\delta_{0}$ is the desired levitation gap, and $r_{d}$ represents the track disturbances. Here the desired levitation gaps of the two levitation units in one module are uniform in steady working condition. Let $I_{i 0}$ denote the steady current, and it obtains (when the terms in the left hand side of (2) are equal to zero)

$$
\begin{aligned}
& I_{10}=\sqrt{\frac{\left(m g+3 N_{1}-N_{2}\right) z_{0}^{2}}{\mu_{0} N^{2} A}}, \\
& I_{20}=\sqrt{\frac{\left(m g+3 N_{2}-N_{1}\right) z_{0}^{2}}{\mu_{0} N^{2} A}} .
\end{aligned}
$$

Given the inductance of the electromagnets, the current loop which adopts a proportional control law to adjust the control voltage for achieving the desired current quickly is given [28]:

$$
u_{i}=k_{c}\left(I_{e i}-I_{i}\right), \quad i=1,2,
$$

where $k_{c}$ is the forward gain of the current loop.

The dynamic model of the module suspension control system with decentralized SISO controllers is determined by (1) (6). On the basis of the stable single suspension control units, the decoupling control scheme will be added to complete the function of decoupling the two levitation 
units of one module. By this way, clear comparisons between the single levitation unit control method and the decoupling module suspension control method can be accomplished. This will be carried out in the following sections.

\section{Decoupling Control Design}

3.1. Decoupling Methodology. In this paper, an adjoint matrix based decoupling control scheme is adopted to provide a simple alternative method for practical control engineers. The module suspension control system is a TITO system, and we consider the TITO process as $Y(s)=G(s) U(s)$ or

$$
\left[\begin{array}{l}
y_{1}(s) \\
y_{2}(s)
\end{array}\right]=\left[\begin{array}{ll}
g_{11}(s) & g_{12}(s) \\
g_{21}(s) & g_{22}(s)
\end{array}\right]\left[\begin{array}{l}
u_{1}(s) \\
u_{2}(s)
\end{array}\right],
$$

where $G(s)$ is strictly proper and stable transfer function and can quantify the proper input-output pairing with diagonal domination.

This section is to design a decoupler $K_{d}(s)$ and a diagonal compensated controller $C(s)$, which guarantees that the resultant closed-loop transfer function is stable and decoupled. The ideal decoupler is the inverse of the transfer function matrix $G^{-1}(s)$. However, the calculation of $G^{-1}(s)$ is too complicated, especially for the system with high dimensions, which make it difficult for practical engineering implementation. In fact, it is clear that the adjoint matrix can be written out easily without much computation burden. Thus, it is more preferable to select the adjoint matrix as the decoupler:

$$
K_{d}(s)=\operatorname{adj} G(s)
$$

As for the module suspension control system, the linearized model around the equilibrium point can be applied to obtain the nominal transfer function matrix. Under the normal working condition, the pitch angle $\theta$ is smaller than $0.5^{\circ}$, so we can suppose that the cosine of $\theta$ equals 1 approximately in (2). The linearized model is given by

$$
\begin{gathered}
-2\left(F_{1}+F_{2}\right)=m_{1}\left(\ddot{\delta}_{1}+\ddot{\delta}_{2}\right) \\
\left(\frac{1}{4} F_{2}-\frac{1}{4} F_{1}\right) l^{2}=J\left(\ddot{\delta}_{1}-\ddot{\delta}_{2}\right) \\
\dot{I}_{i}=\frac{z_{0 i}}{2 k_{e}} u_{i}-\frac{\bar{R} z_{0 i}}{2 k_{e}} I_{i}+\frac{I_{0 i}}{z_{0 i}} \dot{z}_{i} \\
F_{i}=F_{z} z_{i}-F_{I} I_{i} \\
u_{i}=k_{p} k_{c}\left(\delta_{i}-r_{d}\right)+k_{d} k_{c} \dot{\delta}_{i}
\end{gathered}
$$

where $\bar{R}=R+k_{c}, F_{I i}=2 k_{e} I_{i 0} / \delta_{0}^{2}$, and $F_{z i}=2 k_{e} I_{i 0}^{2} / \delta_{0}^{3}$.

During the operation of the maglev train, the track disturbances have the most significant effect on the suspension performance. By omitting the Laplace operator, it gives the transfer function matrix $G(s)$ from $r_{d}$ to $\delta$ with SISO controllers as follows:

$$
\begin{gathered}
G=G_{0}\left(I+G_{0}\right)^{-1}, \\
G_{0}=G_{1} G_{2} G_{5} G_{6}+G_{1} G_{2} G_{4}+G_{1} G_{3},
\end{gathered}
$$

where the meanings of $G_{1 \sim 6}$ can be seen in the Appendix. Hence, the adjoint matrix based decoupler can be rewritten as

$$
K_{d}(s)=\operatorname{adj}\left(G_{0}(s)\left(I+G_{0}(s)\right)^{-1}\right) .
$$

From (11), it is found that the expression of $G_{0}(s)$ is so huge that the calculation of $\left(I+G_{0}(s)\right)^{-1}$ in (12) is still very complicated. Hence, we modify the decoupler as follows:

$$
K_{d}(s)=\left(I+G_{0}(s)\right) \operatorname{adj} G_{0}(s)
$$

In practice, it should be noticed that the format of decoupler $K_{d}(s)$ in (13) makes its calculation simpler, while the coupler $K_{d}(s)$ contains pure integral terms in the denominators. The existence of the pure integral terms in decoupler will demolish the internal stability of the whole system. Therefore, the problem at hand is to improve the decoupler $K_{d}(s)$ such that the pure integral terms can be discarded. Inspired by [29], we make a further modification of the decoupler $K_{d}(s)$ as follows:

$$
K_{d}(s)=\left(I+G_{0}(s)\right) \operatorname{adj}_{0}(s) G_{a}(s)
$$

Here, $G_{a}(s)=\operatorname{diag}\left\{g_{a i}(s)\right\}$ is diagonal stable transfer function matrix, and its elements are designed in the following expression:

$$
g_{a i}(s)=\frac{s^{r_{i}}}{\left(\lambda_{i} s / \omega_{n i}+1\right)^{r_{i}}}, \quad i=1,2
$$

where $r_{i}$ is the maximum order number of the integral terms included in $i$ th column elements of the transfer function $\left(I+G_{0}(s)\right) \operatorname{adj}_{0}(s)$. The choice of $\left(\lambda_{i} s+1\right)^{r_{i}}$ is to ensure that the elements of the decoupler $K_{d}(s)$ are proper and thus physically realizable. Meanwhile, according to the expression of $g_{a i}(s)$, it can be found that the poles brought by $g_{a i}(s)$ will affect the practical dynamic performance of the closed loop system. And the specification of the parameter $\lambda_{i}$ will be discussed in the following procedure according to the desired closed loop performance. When the decoupler $K_{d}(s)$ acts on the original model, the decoupled apparent transfer function matrix is diagonal and is obtained as follows:

$$
Q(s)=G(s) K_{d}(s)=\operatorname{det} G_{0}(s) \cdot G_{a}(s) .
$$

After decoupling, the obtained decoupled transfer function may not satisfy the desired dynamic performance. Even worse, the decoupling may destabilize the whole module suspension control system. Thereby, after the decoupler is determined, an extra diagonal compensated controller is expected to be added to ensure an acceptable dynamic system performance. The block diagram of the proposed decoupling feedback control system is given in Figure 4, which consists of a decoupler $K_{d}(s)$ described in (14) and a diagonal compensated controller $C(s)$.

This scheme is equivalent to a centralized multivariable controller $K(s)$. Once the decoupler $K_{d}(s)$ and the diagonal elements of the compensated controller $C(s)$ are designed, 


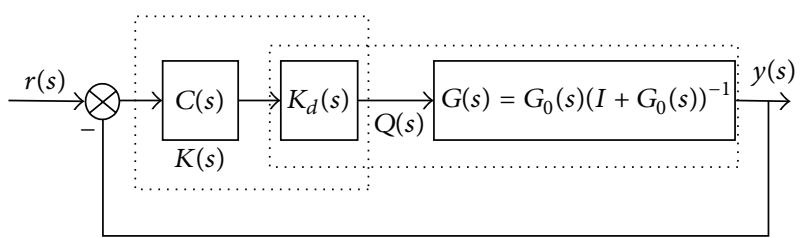

FigURE 4: The block diagram of decoupling feedback control system.

the resultant decoupling controller $K(s)$ is obtained by the following equations:

$$
\begin{gathered}
K(s)=K_{d}(s) C(s), \\
k_{i j}(s)=c_{i i}(s) K_{d_{i j}}(s) \quad i, j=1,2 .
\end{gathered}
$$

According to Figure 4, the closed loop transfer function matrix of the decoupled module suspension system is determined as

$$
\begin{aligned}
H(s) & =G(s) K_{d}(s) C(s)\left[I+G(s) K_{d}(s) C(s)\right]^{-1} \\
& =\operatorname{det} G_{0}(s) G_{a}(s) \cdot C(s)\left[I+\operatorname{det} G_{0}(s) G_{a}(s) \cdot C(s)\right]^{-1} \\
& =\operatorname{diag}\left\{h_{11}(s), h_{22}(s)\right\} .
\end{aligned}
$$

Note that $H(s)$ and $C(s)$ are diagonal. By modifying the expression of (18), the compensated controller $C(s)$ is written as follows:

$$
\begin{aligned}
C(s) & =\left(H(s)^{-1}-I\right)^{-1} \cdot \operatorname{det} G_{0}(s) G_{a}(s) \\
& =\operatorname{diag}\left\{\frac{h_{11}(s)}{\left(1-h_{11}(s)\right) \cdot q_{1}(s)}, \frac{h_{22}(s)}{\left(1-h_{22}(s)\right) \cdot q_{2}(s)}\right\} .
\end{aligned}
$$

From (19), once the desired diagonal elements of the closed loop transfer function matrix $H(s)$ are determined, the elements of the controller $C(s)$ can be ascertained. Without loss of generality, the $i$ th decoupled closed loop transfer function with the undetermined parameters can take the form of [30]

$$
\begin{array}{r}
h_{i i}(s)=\frac{\omega_{n i}^{2}}{\left(s^{2}+2 \omega_{n i} \xi_{i} s+\omega_{n i}^{2}\right)\left(\left(\lambda_{i} / \omega_{n i}\right) s+1\right)^{l_{i}}} \frac{\prod_{k=1}^{m_{i}}\left(s+\bar{z}_{k}\right)}{\prod_{j=1}^{q_{i}}\left(s+p_{j}\right)}, \\
i=1,2,
\end{array}
$$

where $\lambda_{i}, \xi_{i}$, and $\omega_{n i}$ are the adjustable parameters. Here the explanation of the parts of (20) is given. Usually, the conception of dominant poles is used to specify the parameters of high-order control system. The standard 2nd order transfer function locating a pair of conjugation dominant poles in the left half of the complex plane represents the dynamic performance requirement.

The term $\left(\lambda_{i} s / \omega_{n i}+1\right)^{l_{i}}$ emerges here since it guarantees the properness and stability of the decoupler $K_{d}(s)$. To make sure that the elements of resultant centralized controller $K(s)$ are strictly proper and physical realizable, denoting that $r d(G(s))$ is the relative degree of the transfer function $G(s)$, the parameter $l_{i}$ should satisfy the following relationship:

$$
\begin{aligned}
l_{i}=\max \{0, & r d\left(\operatorname{det}\left(G_{0}(s)\right)\right) \\
& -\min \left\{r d\left(G_{0}^{i 1}(s)\right), r d\left(G_{0}^{i 2}(s)\right)\right\} \\
& \left.+r_{i}+m_{i}-q_{i}-1\right\}, \quad i=1,2 .
\end{aligned}
$$

The parameter $\lambda_{i}$ has an effect on the bandwidth and the stability margin of the $i$ th loop transfer function and also decides the cut-off frequency in high frequency domain. Though we can specify the parameter $\lambda_{i}$ to be arbitrarily small such that the pole brought by $\left(\lambda_{i} s / \omega_{n i}+1\right)^{l_{i}}$ can be far away from the dominant poles, it will result in a bigger overshot in dynamic performance as well.

At last, the residual part of $h_{i i}(s)$ represents the inherent characteristic of the original process obtained by stable pole-zero cancellation. According to the analysis procedure above, the decoupler $K_{d}(s)$ can be obtained by (14), and the compensated controller $C(s)$ is determined based on (19) when the desired diagonal elements of the closed loop transfer function matrix $H(s)$ are given. Then, the resultant controller $K(s)$ is obtained by (17). Though the proposed decoupling design is based on TITO system, it also provides an alternative solution to unstable MIMO system with high dimensions.

3.2. Practical Considerations on Controllers and Robust Stability Analysis. Following the procedure given above, the decoupler $K_{d}(s)$ is obtained to decouple the module suspension system to two SISO loops, and the compensated controller $C(s)$ is to guarantee the stability and performance of each loop. However, we adopted the linearized model as the nominal system in the procedure of the decoupling analysis. Various sources of disturbances and unmodeled dynamics and nonlinearities may cause parametric uncertainties in the practical control system. Hence, the specifications of the controller parameters should take the robustness to parametric uncertainties and set-point tracking capacity into consideration. The common criterion to measure the robustness to process uncertainties is the maximum sensitivity. The output sensitivity function and the complementary sensitivity function are defined as follows:

$$
\begin{gathered}
S(s)=(I+G(s) K(s))^{-1}, \\
T(s)=G(s) K(s)(I+G(s) K(s))^{-1} .
\end{gathered}
$$

It is obvious that $S(s)$ is diagonal, and for the $i$ th loop, we can use

$$
M_{\mathrm{st}_{i}}=\max _{\omega}\left(\left|S_{i i}(j \omega)\right|,\left|T_{i i}(j \omega)\right|\right), \quad i=1,2 \forall \omega \in R
$$

as the robustness index [31]. The reasonable values of $M_{s}$ are in the range between 1.2 and 2.0. A smaller value of $M_{s}$ is preferred to guarantee the robustness. Actually, robustness is 
usually achieved at the expense of system performance in the normal working condition, which implies that compromise between robustness and system performance has to be found in practical application.

For the magnetic suspension system, rapid response to track disturbance is crucial for good dynamic performance, which means that smaller overshoot and setting time are demanded. In this paper, the system performance of the control system is evaluated by calculating the integrated absolute error (IAE) due to a unit step load disturbance. The IAE index for the $i$ th loop is defined as

$$
\mathrm{IAE}=\int_{0}^{\infty}\left|r_{i}(t)-y_{i}(t)\right| d t, \quad i=1,2
$$

The controller with smaller IAE is considered to have a better control performance here.

From the decoupling design procedure, the controller $K(s)$ is determined by three parameters $\lambda_{i}, \xi_{i}$, and $\omega_{n i}$. The determination of those parameters is based on the trade-off between robustness and system performance. In this work, plots are introduced to show the relationship between system performance and robustness in regard to the controller parameters, which will be illustrated in Section 4.1.

The decoupling design for the module suspension system is given in Section 3.1, and it is obvious that the orders of the elements of the resultant controller $K(s)$ are so high. Hence, it is difficult to implement the controller in physics. In engineering practice, it is an advisable way to solve this problem by approximating their elements with a reducedorder model. However, it is difficult to obtain a perfect reduced-order controller which matches the original one well in the whole frequency domain. In practice, we can require that the reduced controller matches the original one in a desired domain of $s \in D_{\omega i} \triangleq\left\{s \in C \mid s=j \omega, \omega_{s i} \leq \omega \leq \omega_{m i}\right\}$. The value of $\omega_{m i}$ should be well chosen to be large enough such that it is well beyond the system bandwidth [31]. In this paper, the frequency domain is chosen as the values between $\omega_{m i}=\omega_{q i} / 10$ and $\omega_{m i}=10 \omega_{q i}$, and $\omega_{q i}$ is the $0 \mathrm{db}$ crossover frequency of the $i$ th loop transfer function $q_{i}$. The elements of the resultant decoupled controller $K(s)$ have the following format:

$$
K_{i j}(s)=A(s)=\frac{b_{m} s^{m}+\cdots+b_{1} s+A(0)}{a_{n} s^{n}+a_{n-1} s^{n-1}+\cdots+a_{1} s+1} .
$$

Assume that the frequency response $A\left(j w_{k}\right)$ can be approximated by a reduced-order model $A_{r}(s)$ with orders $\gamma$ and $k$ in the numerator and denominator, respectively. The approximation rational transfer function is described by (26) with identical stationary gain:

$$
A_{r}(s)=\frac{N(s)}{D(s)}=\frac{\beta_{r} s^{r}+\cdots+\beta_{1} s+A(0)}{\alpha_{k} s^{k}+\alpha_{k-1} s^{k-1}+\cdots+\alpha_{1} s+1}
$$

Denoting that $\boldsymbol{\theta}=\left(\alpha_{1}, \ldots, \alpha_{k}, \beta_{1}, \ldots, \beta_{r}\right)$ is the undetermined parameters vector, the proposed model approximation method is to search the optimal parameters to satisfy the following objective function $J[32,33]$ :

$$
\begin{gathered}
J=\min _{s \in D_{\omega i}} \frac{1}{N} \sum_{k=1}^{N}\left[\left|W\left(j w_{k}\right)\right|^{2} \cdot\left|A\left(j w_{k}\right)-A_{r}\left(j w_{k}, \boldsymbol{\theta}\right)\right|^{2}\right], \\
W\left(j w_{k}\right)=\left|\frac{D\left(j w_{k}, \alpha^{i}\right)}{D\left(j w_{k}, \alpha^{i-1}\right)}\right|,
\end{gathered}
$$

where $W\left(j w_{k}\right)$ is a weighting function. From the above, when the order of the approximation model is chosen, the parameterization for the reduced-order model $A_{r}(s)$ is determined if the mean squared error is under preestablished tolerance.

After the model reduction, the resultant controller $K(s)$ may have any physically realizable structure. PID structure is one of the first developed control strategies with simple structure and well-known tuning rules, which makes it maintain dominance in practicing engineering applications for several decades. Though more advance control algorithms have been developed, the PID controllers are always preferred unless they do not give satisfactory performance [33]. Hence, in this work, the resultant controller $K(s)$ is approximated by PID controllers. A pure differential term in PID controller will cause infinite high frequency gain. Also, it is undesirable and impossible to realize such a controller. In view of that, a second order low-pass filter is introduced to replace the common procedure of the pure differential terms in the PID controller. The second order low-pass filter is shown as follows:

$$
K_{f}(s)=\frac{1}{1+s T_{f}+s^{2}\left(T_{f}^{2} / N\right)}
$$

Then, the PID controller structure used in this paper is written as

$$
\begin{aligned}
K_{\mathrm{PID}}(s) & =K_{f}(s)\left(K_{p}+\frac{T_{i}}{s}+T_{d} s\right) \\
& =\frac{T_{d} s^{2}+K_{p} s+T_{i}}{s\left(1+s T_{f}+s^{2}\left(T_{f}^{2} / N\right)\right)},
\end{aligned}
$$

where $K_{P}$ is the proportional gain, $T_{i}$ is the integration constant, $K_{d}$ is the derivative constant, and $T_{F}$ is the derivative filter constant. The PID controller is obtained based on the model reduction method mentioned above. Basically, the elements of the resultant controller $K(s)$ contain integrator to eliminate the stationary error due to setpoint or load changes. For simplicity, this paper denotes $K(s)=P(s) / s$ by removing the integrator. Applying the approximation method to the inverse of $P(s)$, the approximation model can be obtained as follows:

$$
P(s) \approx \frac{\beta_{2} s^{2}+\beta_{1} s+P(0)}{\alpha_{2} s^{2}+\alpha_{1} s+1}
$$


According to (29) and (30), the PID controller parameters are given as follows:

$$
\begin{gathered}
K_{p}=\beta_{1}, \quad T_{i}=P(0), \quad T_{d}=\beta_{2}, \\
T_{f}=\alpha_{1}, \quad N=\frac{\alpha_{1}^{2}}{\alpha_{2}} .
\end{gathered}
$$

Since an approximation is adopted to transform the ideal decoupling to the PID structure, the robust stability needs to be checked when uncertainties exist in the model of the controlled levitation module. Assume that the practical module suspension control system has an multiplicative uncertainty compared with the linearized model $G(s)$; then, it is presented as

$$
G_{p}(s)=G(s)(I+\Delta(s)) .
$$

A rational weighting function which represents the uncertainties of the module suspension control system is given as follows:

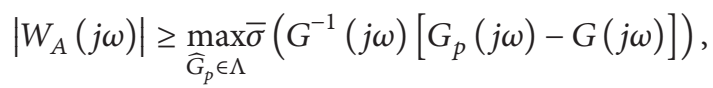

where $\Lambda$ is a set containing all possible real model $G_{p}(s)$. Hence, the robust stability criterion for the close loop system is obtained as [34]

$$
\begin{gathered}
\bar{\sigma}(M(j \omega))<1, \quad \forall \omega \in[0, \infty), \\
M(s)=-W_{A}(s) K_{\mathrm{PID}}(s)\left[I+K_{\mathrm{PID}}(s) G(s)\right]^{-1} .
\end{gathered}
$$

In this work, the weighting function of the system uncertainties is obtained by frequency sweeping experiments on the full-scale single bogie of CMS-04 maglev train. The expression of the weighting function is given in Section 4.2. By this way, we can calculate and plot the left hand side of (34) and then compare it with the unity to see whether robust stability is satisfied.

\section{Case Study}

4.1. Parameters Specification. This section gives parameters specification procedure of the resultant decoupling controller $K(s)$. The related calculation is based on the actual parameters of the CMS04 low speed maglev train which are shown in Table 1 .

According to the design procedure of the decoupler $K_{d}(s)$, it is not necessary to calculate the transfer function matrix of the module suspension control system $G(s)$. Instead, the transfer function matrix $G_{0}(s)$ is obtained by using the parameters listed in Table 1:

$$
G_{0}(s)=\left[\begin{array}{cc}
\frac{68271.3(s+40.15)}{s^{2}(s+1135)} & \frac{553.29(s-3285)}{s^{2}(s+1135)} \\
\frac{553.29(s-3285)}{s^{2}(s+1135)} & \frac{68271.3(s+40.15)}{s^{2}(s+1135)}
\end{array}\right] .
$$

TABLE 1: Parameters of the module suspension control system.

\begin{tabular}{lc}
\hline Property & Value \\
\hline$m$ & $1020 \mathrm{~kg}$ \\
$N_{1}, N_{2}$ & $10 \mathrm{KN}$ \\
$A$ & $0.0186 \mathrm{~m}^{2}$ \\
$l$ & $2.65 \mathrm{~m}$ \\
$J$ & $910 \mathrm{~kg} \cdot \mathrm{m}^{2}$ \\
$N$ & 320 \\
$k_{c}$ & 300 \\
$\mu_{0}$ & $4 \pi$ \\
$k_{p}$ & 40000 \\
$k_{d}$ & 33 \\
$\delta_{0}$ & $9 \mathrm{~mm}$ \\
$R$ & $1.0 \Omega$ \\
\hline
\end{tabular}

From the expression of $G_{0}(s)$, it is seen that the nondiagonal elements have one nonminimum phase zero. So the ideal inverted decoupling method may lead to RHP poles in the decoupler. This can be avoided by using the decoupler given in (14). The decoupler is calculated as follows:

$$
\begin{aligned}
K_{d}(s) & =\left(I+G_{0}(s)\right) \operatorname{adj} G_{0}(s) G_{a}(s) \\
& =\left[\begin{array}{ll}
k d_{11} & k d_{12} \\
k d_{21} & k d_{22}
\end{array}\right],
\end{aligned}
$$

$k d_{11}$

$$
\begin{gathered}
=\frac{68271.3 s^{r_{1}}(s+1074)(s+15.24)\left(s^{2}+86.04 s+3768\right)}{s^{4}(s+1135)^{2}\left(\lambda_{1} s / \omega_{n 1}+1\right)^{r_{1}}}, \\
k d_{12}=\frac{-553.29 s^{r_{1}}(s-3285)}{s^{2}(s+1135)\left(\lambda_{2} s / \omega_{n 2}+1\right)^{r_{2}}}, \\
k d_{21}=\frac{-553.29 s^{r_{1}}(s-3285)}{s^{2}(s+1135)\left(\lambda_{1} s / \omega_{n 2}+1\right)^{r_{1}}},
\end{gathered}
$$

$$
\begin{aligned}
& k d_{22} \\
& =\frac{68271.3 s^{r_{2}}(s+1074)(s+15.24)\left(s^{2}+86.04 s+3768\right)}{s^{4}(s+1135)^{2}\left(\lambda_{2} s / \omega_{n 2}+1\right)^{r_{2}}} .
\end{aligned}
$$

Since the maximum order numbers of the integral terms included in the column elements of the transfer function ( $I+$ $\left.G_{0}(s)\right) \operatorname{adj} G_{0}(s)$ are both 4 , we choose $r_{1}=r_{2}=4$. After the decoupler $K_{d}(s)$ is determined, the apparent transfer function matrix of the module suspension control system is decoupled to be two SISO loops, and its elements $q_{i}(s)$ can be given as follows:

$$
q_{i}(s)=\frac{4.66 \times 10^{9}\left(s^{2}+80.72 s+902.6\right)}{(s+1135)^{2}\left(\lambda_{i} s / \omega_{n i}+1\right)^{4}}, \quad i=1,2 .
$$

It shows that the zeros and poles of $q_{i}(s)$ are in the left side of the complex plane. To simplify the structure of 


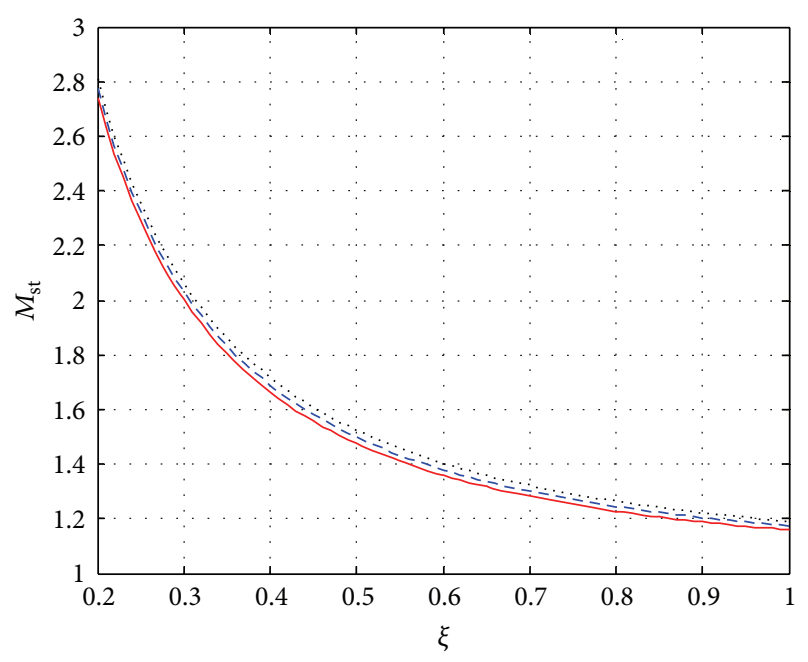

(a) Plots of robustness index $\left(M_{\text {st }}\right)$

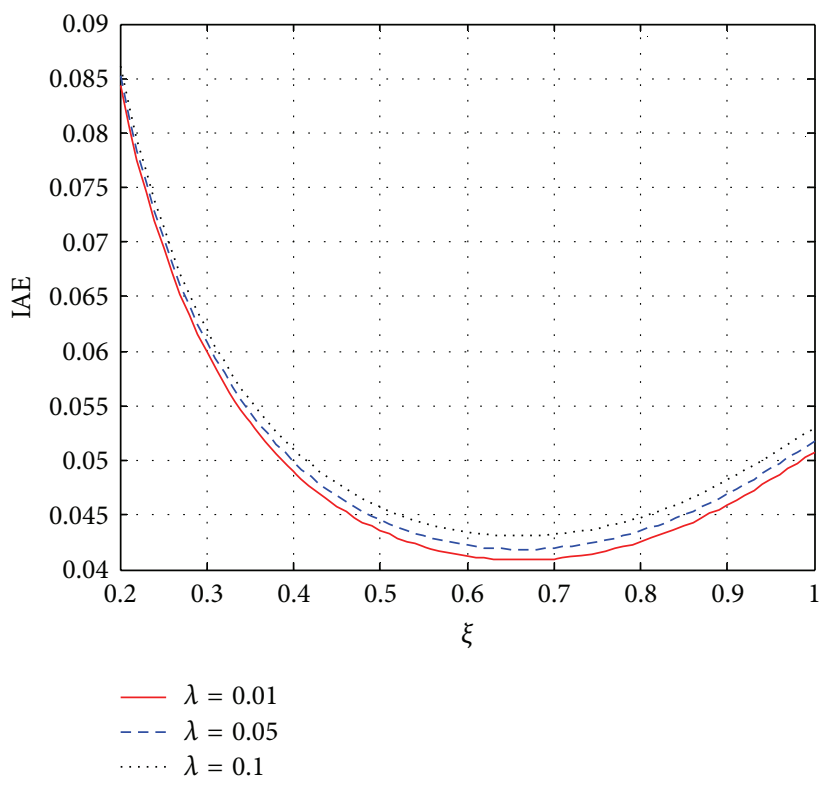

(b) Plots of performance index (IEA)

Figure 5: Robustness index $\left(M_{\mathrm{st}}\right)$ and performance index as functions of the parameters $\xi$ and $\lambda$.

closed loop transfer function $H(s)$, those dynamics can be discarded by stable pole-zero cancellation. According to (21), the parameter $l_{i}$ is chosen to be 1 . Hence, the compensated controller $C(s)$ is given as follows:

$$
\begin{aligned}
c_{i i}(s)= & \frac{\omega_{n i}^{2}}{\left(s^{2}+2 \omega_{n i} \xi_{i} s+\omega_{n i}^{2}\right)\left(\lambda_{i} s / \omega_{n i}+1\right)-\omega_{n i}^{2}} \\
& \cdot \frac{(s+1135)^{2}\left(\lambda_{i} s / \omega_{n i}+1\right)^{4}}{4.66 \times 10^{9}\left(s^{2}+80.72 s+902.6\right)}, \quad i=1,2 .
\end{aligned}
$$

Then, the resultant controller $K(s)$ is obtained through (17). However, the three controller parameters $\lambda_{i}$, $\xi_{i}$, and $\omega_{n i}$ are still not determined. So, the work at hand is to analyze the relationship among the controller parameters and the indexes of robustness and system performance, which helps us to specify the controller parameters. Normally, the levitated units of one module are supposed to have the same performance, so the two diagonal elements of the compensated controller $C(s)$ are equal. Here, the subscript of the parameters is discarded in the following parameters specification procedure. In Section 3.2, we choose $M_{\text {st }}$ and IAE as the evaluation indexes of robustness and system performance, respectively. The situation of three controller parameters is more complicated because it needs three dimensions plots to express the relationships. Through the multiple plots, we find that the variation of $\omega_{n}$ does not affect the robustness index $M_{\mathrm{st}}$ described in (23), so we give the plots of robustness index $\left(M_{\mathrm{st}}\right)$ and performance index (IAE) as functions of the parameters $\xi$ and $\lambda$ by setting $\omega_{n}$ as a constant firstly.

Figure 5(a) shows the plots of robustness index $\left(M_{\mathrm{st}}\right)$ as function of the parameters $\xi$ and $\lambda$, and Figure 5(b) shows the plots of performance index (IAE) as function of the parameters $\xi$ and $\lambda$. Figure 5(a) shows that the robustness index $M_{\text {st }}$ decreases when the parameter $\xi$ increases. When $\xi$ is bigger than almost $0.3, M_{\text {st }}$ will be in the reasonable range between 1.2 and 2.0. Besides, Figure 5(b) indicates that the minimization of IAE will be obtained when $\xi$ is 0.66 , which also gives acceptable robustness. For the correlation among IAE, $M_{\mathrm{st}}$, and $\lambda$, IAE and $M_{\mathrm{st}}$ both decrease when the parameter $\lambda$ decreases. Once we choose $\xi$ as 0.66 , a smaller value of $\lambda$ means a promotion of both the performance and the robustness.

Next, we give the plots of robustness index $\left(M_{\mathrm{st}}\right)$ and performance index (IAE) as functions of the parameters $\omega_{n}$ and $\lambda$ in Figures 6(a) and 6(b) when $\xi$ is 0.66 . The plots in Figure 6(a) show that the level curve of $M_{\mathrm{st}}$ is horizontal, which means that robustness index will not be influenced by the variation of the parameter $\omega_{n}$. Figure $6(\mathrm{~b})$ gives the conclusion that the performance index (IAE) decreases when the parameter $\omega_{n}$ increases. Just like Figure 5, though the influence caused by the parameter $\lambda$ is much smaller, the plots also indicate that robustness index $M_{\text {st }}$ and performance index (IAE) will be smaller by setting $\lambda$ on a lower value when the parameters $\xi$ and $\omega_{n}$ are fixed. Actually, the peak time is also important for the suspension control system. The mentioned current loop method above is to shorten the response time of the electromagnets. It is not expected that the decoupling design destroys the original intention of the current loop. So the specification of the parameters must satisfy the demand of the peak time. Besides, it is obvious that a smaller $\lambda$ and a bigger $\omega_{n}$ can both benefit the robustness and performance, while it also give a higher control gain. For the measurement noise, high gain means too large control actions. So a natural way to solve this problem is to choose acceptable values of $\lambda$ and $\omega_{n}$ by considering the measurement noise. 


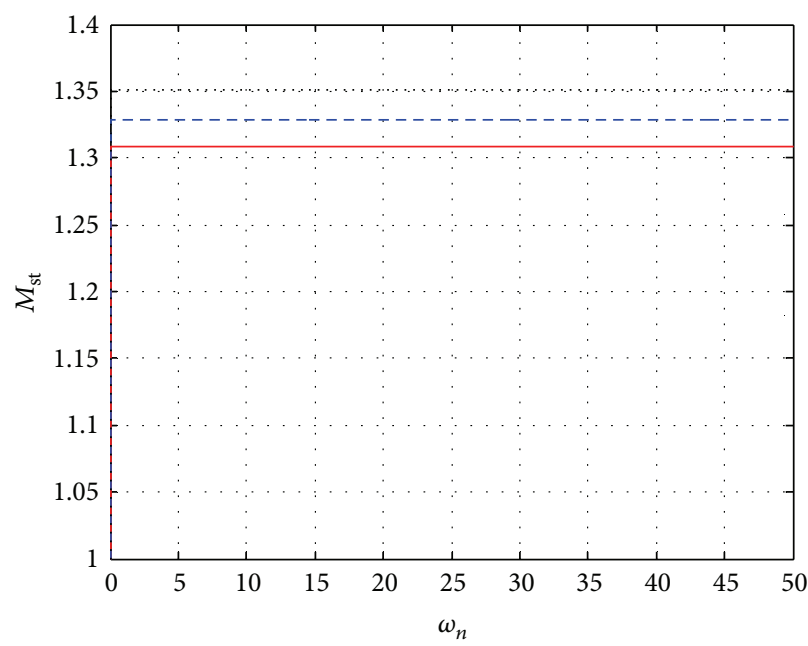

(a) Plots of robustness index $\left(M_{\mathrm{st}}\right)$

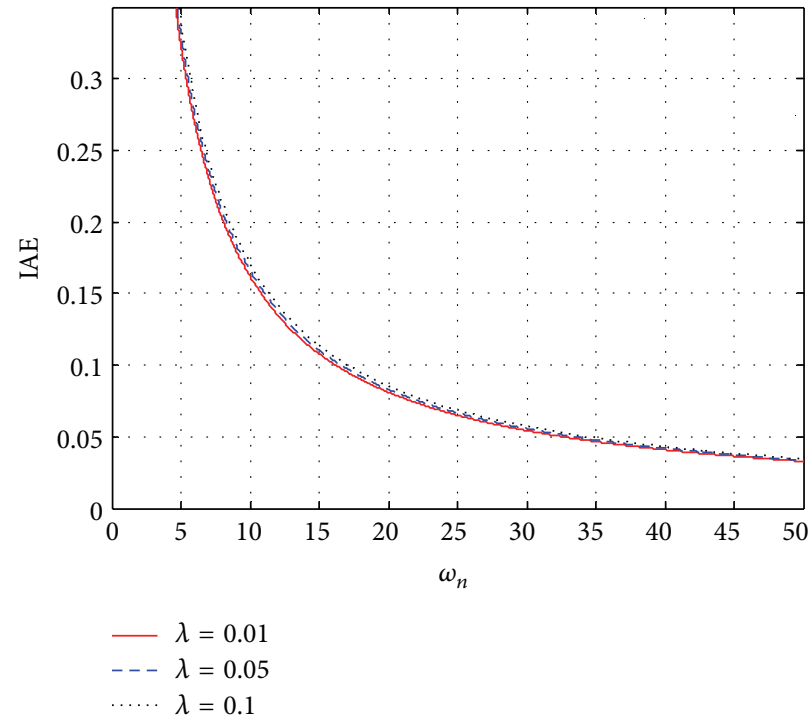

(b) Plots of performance index (IEA)

FIgURE 6: Robustness index $\left(M_{\mathrm{st}}\right)$ and performance index (IAE) as functions of the parameters $\omega_{n}$ and $\lambda$.

In brief, from the plots which present the relationship among the robustness index, performance index, and the three controller parameters, we determine the parameters $\lambda$, $\xi$, and $\omega_{n}$ as follows.

(1) The parameter $\xi=0.66$ gives an acceptable robustness as well as minimization of IAE.

(2) The parameter $\omega_{n}$ does not affect the robustness index $M_{\mathrm{st}}$. It is only determined by the trade-off between the peak time and acceptable control gain for the measurement noise. In practical physical controller, the filter circuit is already designed to make the measurement noise in an acceptable degree. Denote that the peak time $t_{r}<0.1 \mathrm{~s}$; then, we get $\omega_{n}>40$. Though a much bigger value of $\omega_{n}$ can make the peak time even smaller, the induced high control gain will amplify actuator actions caused by the measurement noise. So we choose the parameter $\omega_{n}$ as 40 .

(3) Once the parameters of the standard 2nd order system are specified, the parameter $\lambda_{i}$ can serve as a monotonously tuning parameter to affect dynamic performance and robustness. In this paper, we choose the desired robustness value $M_{\mathrm{st}}=1.33$, and then $\lambda$ will be 0.05 .

According to the parameters specified above, the robustness index is $M_{\mathrm{st}}=1.33$, and the minimization of IAE is 0.046 . The resultant centralized controller $K_{\mathrm{PID}}(s)$ is given in Table 2.

4.2. Simulations and Experimental Results. To verify the decoupling effect and the performance, the closed loop responses of the nominal control system subject to a $1 \mathrm{~mm}$ square wave are shown in Figure 7. The initial condition is
TABLE 2: PID parameters of the controller $K_{\mathrm{PID}}(s)$.

\begin{tabular}{lccccc}
\hline \multirow{2}{*}{ Location } & \multicolumn{5}{c}{ PID parameters } \\
& $K_{p}$ & $T_{i}$ & $T_{d}$ & $N$ & $T_{f}$ \\
\hline $1-1 / 2-2$ & 3.91 & 43.8 & 0.0743 & 4.927 & 0.1024 \\
$1-2 / 2-1$ & $4.29 \times 10^{-6}$ & 0 & 0.0215 & 4.927 & 0.1024 \\
\hline
\end{tabular}

that the module levitates at a gap of $9 \mathrm{~mm}$ and the square wave disturbance is added at $t=0 \mathrm{~s}$ in the first loop and at $t=1 \mathrm{~s}$ in the second loop. In the ideal case, the dynamics of the two levitated units is decoupled perfectly with the designed high order centralized controller $K(s)$. Compared with the system responses generated by traditional decentralized controllers, the approximated centralized controller with PID formula can provide a better decoupling performance. Meanwhile, the peak time is smaller than the desired value of $0.1 \mathrm{~s}$ and a lower overshoot is also obtained. Besides, a gap disturbance with a magnitude of $1 \mathrm{~mm}$ is added in the first loop to show the disturbance rejection performance at $t=2.5 \mathrm{~s}$. And the system responses are also shown in Figure 7. It can be seen that the disturbance rejection performance is dramatically improved with the approximated centralized PID controller.

In practice, the carrying mass changes apparently when passengers get on/off. Hence, simulations are also made when the mass of the maglev train changes. Here, it is assumed that the steady current of electromagnets can response to the load change rapidly, which is already realized in CMS0 4 maglev train. And the results show that it affects the decoupling of the levitation units of the module in a very low degree. Besides, under the ideal working condition, the mass of the carriage is transferred to each levitation unit through air spring on average. However, more or less difference will exist due to the installation error in practice, which causes directly that 


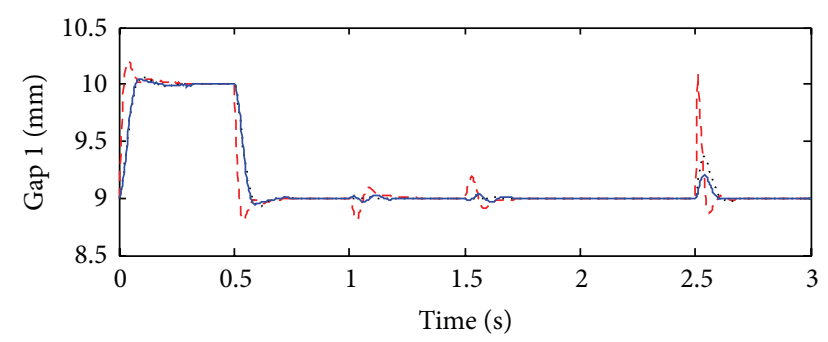

(a)

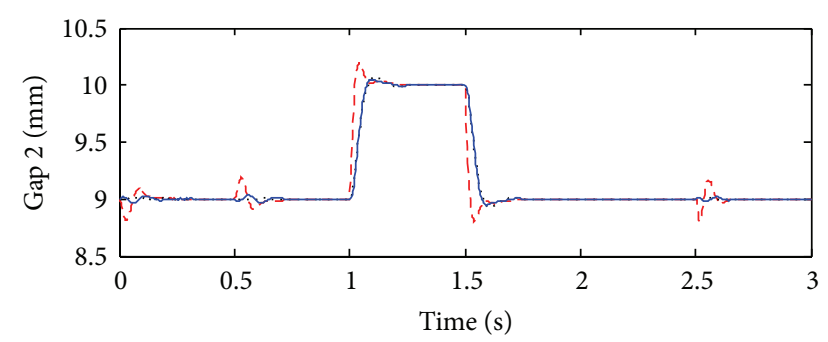

-- - Nondecoupling

..... Ideal decoupling

— PID decoupling

(b)

FIGURE 7: Closed loop responses of the nominal linearized control system.

the steady currents of the two levitation units in one module are different. So we give simulations by assuming that the transformed force on the levitation unit one is $1 \mathrm{KN}$ less than that of the others, and the simulation results are shown in Figure 8. The results demonstrate that a good decoupling performance can still be obtained. Meanwhile, the system responses and disturbance rejection performance can also be guaranteed.

To evaluate the robust stability of the proposed controllers, experiments have been performed on the full-scale single bogie of CMS-04 maglev train to obtain the weighting function of the system uncertainties in this work. The picture of the full-scale single bogie of CMS-04 maglev train is shown in Figure 9.

Firstly, we adopted the traditional decentralized controllers to make the module levitate steadily. Then a sine external excitation is introduced into one of the levitation points in variable frequencies. The outputs under variable frequencies represent the frequency response of the real suspension control system model $G_{p}(s)$. In the experiments, the frequency domain is chosen from $0.1 \mathrm{~Hz}$ to $50 \mathrm{~Hz}$. Many repetitive experiments have been finished under the considerations such as different steady operation points and different tracks. Through those experimental results, the possible set of real model $\Lambda$ is obtained. Because the two levitation units are symmetrical, here the weighting function from multiple experiments is given as follows:

$$
W_{A}(s)=\frac{0.0318 s+0.4}{(0.0318 / 0.6) s+1} \cdot I
$$

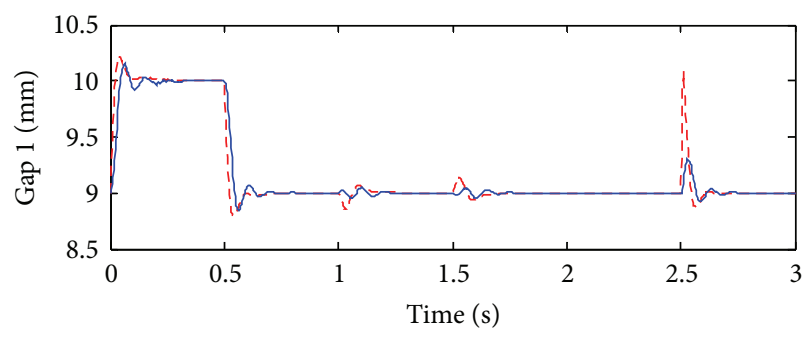

(a)

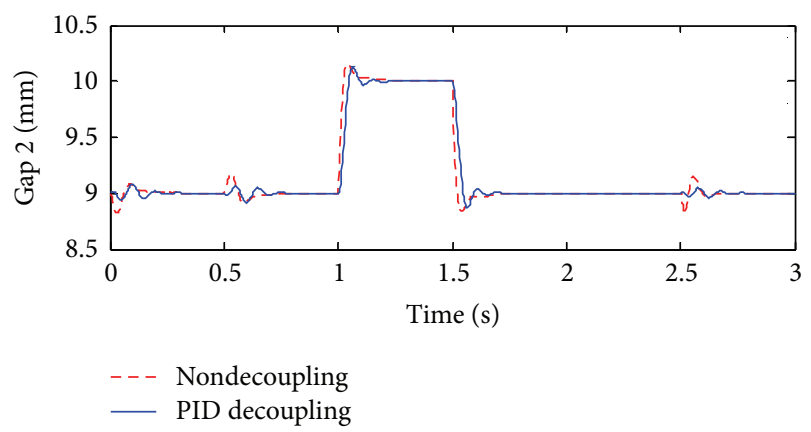

(b)

FIGURE 8: Closed loop responses of the nominal linearized control system when the transformed forces is different.

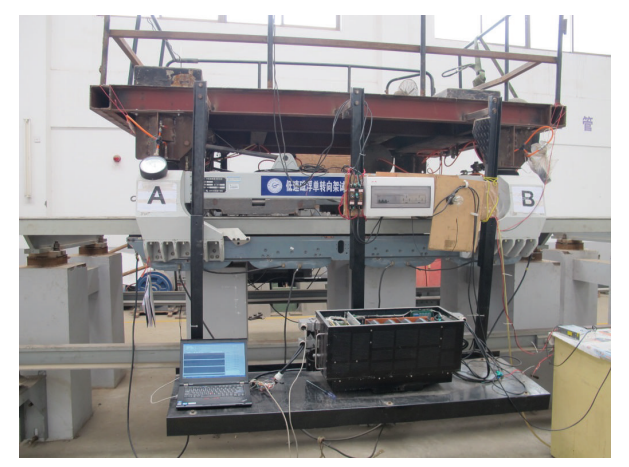

FIGURE 9: Full-scale single bogie of CMS-04 maglev train.

The weighting function $W_{A}(s)$ can be loosely interpreted as the input of each levitation unit has almost $40 \%$ uncertainty in the low frequency domain and up to $60 \%$ uncertainty at the high frequencies. The magnitude plot of the maximum singular value of $M(s)$ is shown in Figure 10. It can be seen that the peak value of $\bar{\sigma}(M(j \omega))$ is much less than the unity, which indicates that the proposed controller design indeed provides good robust stability.

To validate the practice effect of the proposed decoupling method, some experiments are also executed on the full-scale single bogie of CMS-0 4 maglev train. Firstly, we still test the decoupling capacity when $1 \mathrm{~mm}$ square wave disturbance is added. In the first stage, the module is levitated steadily around the operation point $\delta_{0}=9 \mathrm{~mm}$ with the designed decoupling controller. Then, a $1 \mathrm{~mm}$ square wave disturbance lasting $2 \mathrm{~s}$ is added to the levitation gap of levitation unit one. The curves of the levitation gap and the current are shown in Figure 11. For comparison, the same experiments 


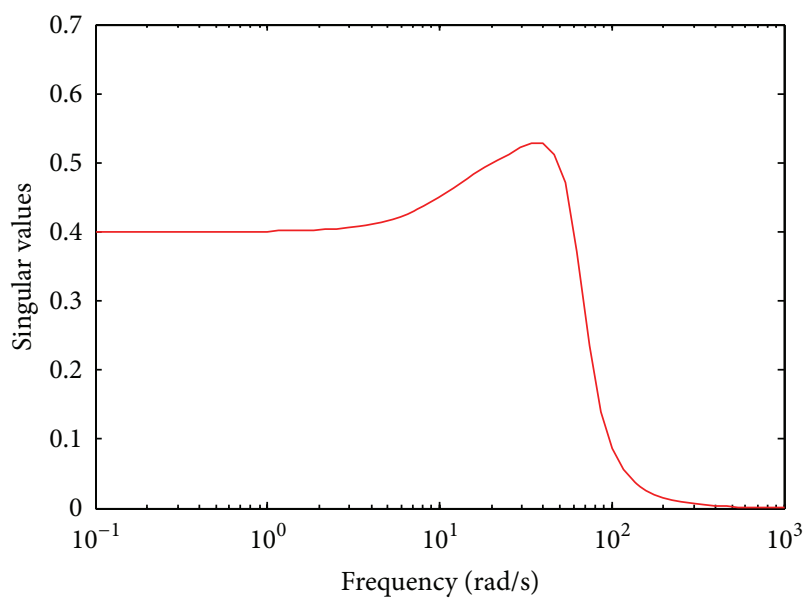

FIgURE 10: The maximum singular value of $M(s)$.

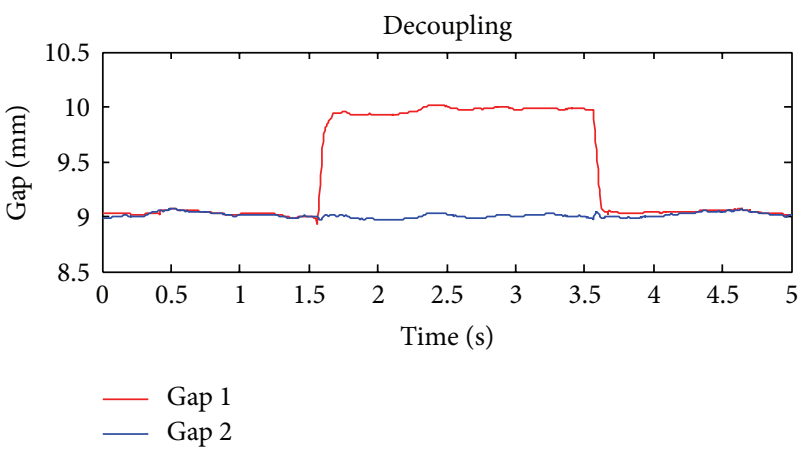

(a)

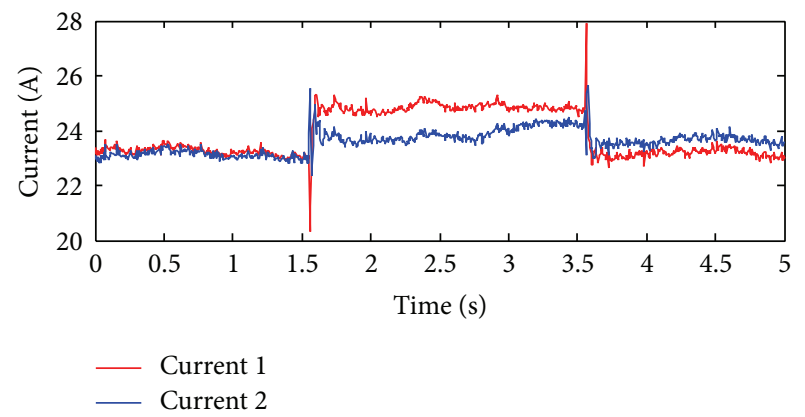

(b)

FIGURE 11: The response of the levitation gaps and the current when decoupling design is adopted.

with traditional SISO controllers are also carried out, and the responses of the levitation gap and the current are shown in Figure 12.

When the module suspension system is controlled with the traditional SISO controllers, it is seen that the gap curve of the levitation unit two fluctuates to almost $0.2 \mathrm{~mm}$ when the square wave disturbance is added into the levitation unit one. From the experiment curve with the proposed decoupling design, the gap of the levitation unit two just varies less than $0.05 \mathrm{~mm}$, which shows that the decoupling design reduces the coupling between the two levitation units by $75 \%$. Because

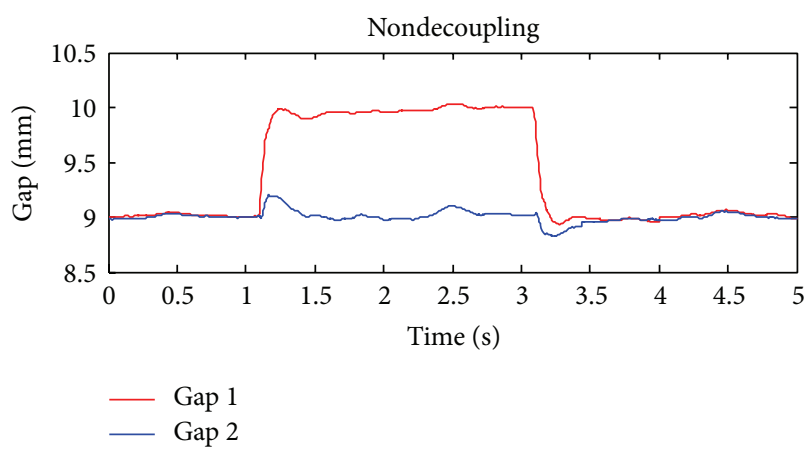

(a)

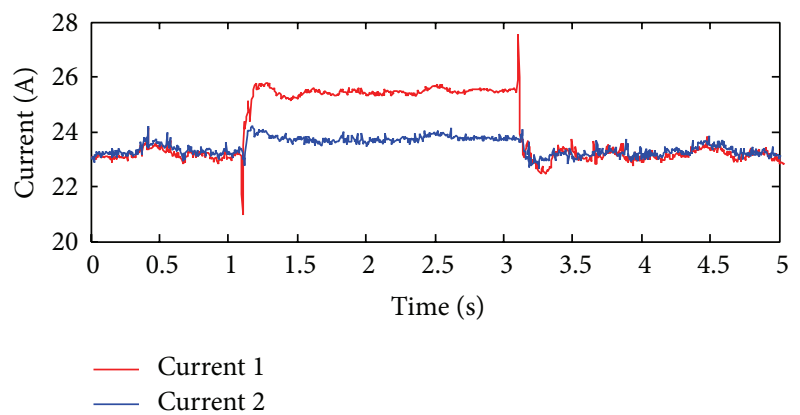

(b)

FIGURE 12: The response of the levitation gaps and the current when traditional SISO controllers are adopted.

the decoupling controller can receive the sensor message of both levitation units, the current of levitation unit two can be adjusted accordingly so that the fluctuation of the levitation unit two is suppressed. From the experimental results shown in Figure 11, it can be concluded that the proposed decoupling control design can provide a good decoupling performance.

Besides, experiments assuming that one levitation unit of the module breaks down are also carried out. In the experiments, we give a huge disturbance to make the levitation unit one collapse artificially and simultaneously record the dynamic response of the levitation gap of the levitation unit two. The curves of the levitation gap are shown in Figures 13 and 14. In Figure 13, when the levitation unit one breaks down, the large gravity action will also destabilize the levitation unit two because of the coupling between the two levitation units. Meanwhile, the violent collision against the tracks will last a long time until the module starts to levitate again. However, when the decoupling design in this paper is used in the suspension control of the module, the levitation unit two can be still stable after a short adjustment though the levitation unit one is destabilized artificially. In Figure 14, the levitation unit one can start to levitate in a short time. And the long time of the levitation procedure turns up because a slow levitation technology is adopted in the controller to make the levitation procedure more comfortable.

\section{Conclusions}

In the paper, an engineering oriented decoupling controller design has been proposed for the module suspension control 


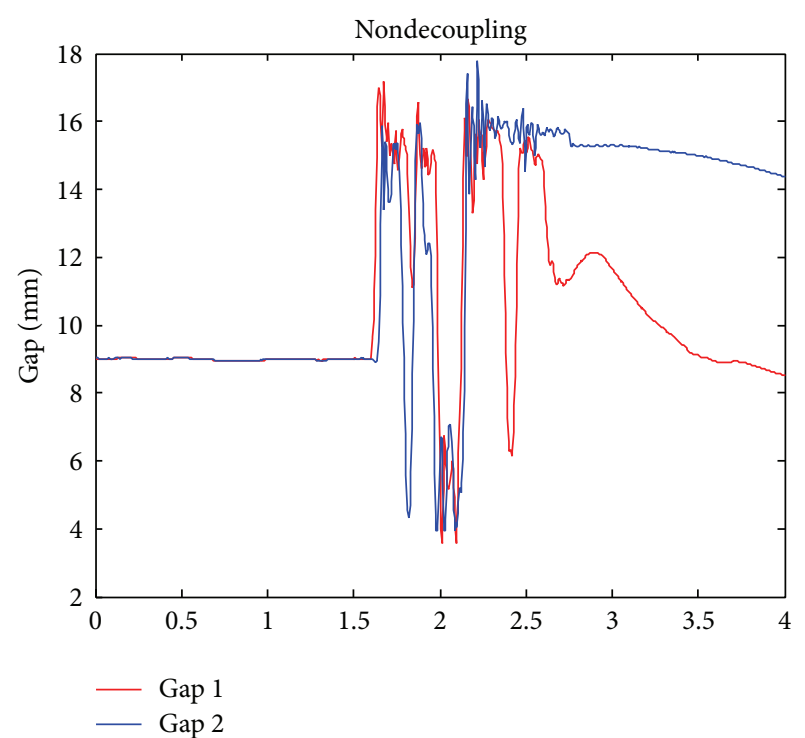

FIGURE 13: The response of the levitation gaps with traditional SISO controllers when levitation unit one breaks down.

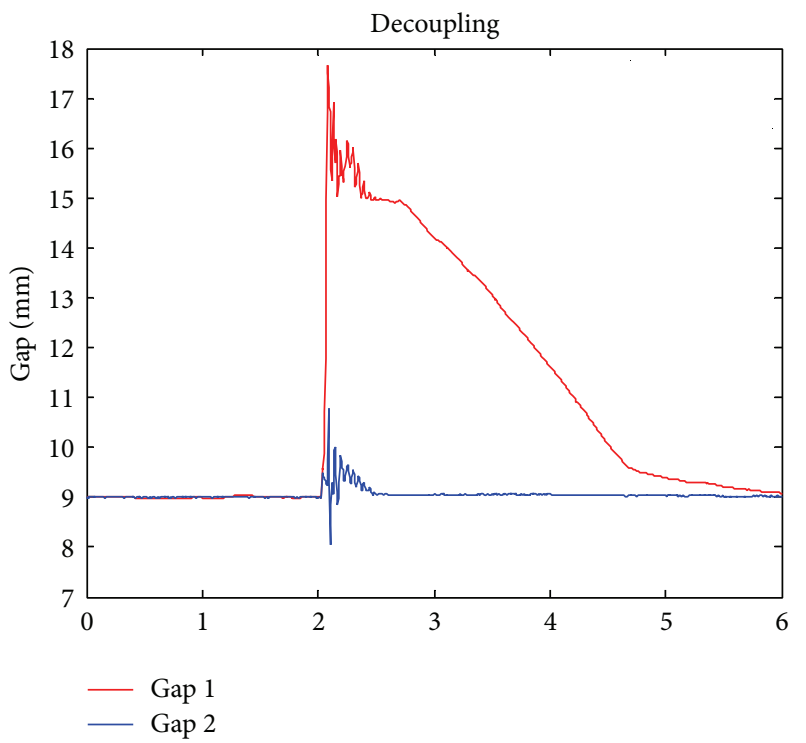

Figure 14: The response of the levitation gaps with decoupling controllers when levitation unit one breaks down.

system. A modified adjoint transfer matrix based decoupler is used to make the system transfer matrix be diagonal and then the compensated controller is designed individually according to the desired close loop system performance. The resultant controller parameters have been determined by the trade-offs between the performance index and robustness index. To avoid high order of the resultant controller, model reduction method is adopted to simplify it to be a multivariable PID controller. Simulations are accomplished to show that the designed controller has a good decoupling performance and system response. Due to the fact that the linear model cannot include the uncertainties caused by nonlinearities and other reasons, an experimental method is used to calculate the weighting function of the system uncertainties, based on which the robust stability of the proposed controllers has been checked. Finally, experiments on the full-scale single bogie of CMS-04 maglev train have been executed to verify the effectiveness of the proposed approach in practical physical model. Compared with the experiment results using the traditional SISO controllers, the presented decoupling design can provide satisfactory decoupling and set-point tracking performance.

\section{Appendix}

The meanings of $G_{i}, i=1 \sim 6$ are shown as follows:

$$
\begin{aligned}
& G_{1}=\left[\begin{array}{cc}
-\frac{1}{m_{1}}-\frac{l^{2}}{8 J} & -\frac{1}{m_{1}}+\frac{l^{2}}{8 J} \\
-\frac{1}{m_{1}}+\frac{l^{2}}{8 J} & -\frac{1}{m_{1}}-\frac{l^{2}}{8 J}
\end{array}\right], \\
& G_{2}=\left[\begin{array}{cc}
-F_{I 1} & 0 \\
0 & -F_{I 2}
\end{array}\right] \text {, } \\
& G_{3}=\left[\begin{array}{cc}
\frac{3 F_{z 1}}{4} & \frac{F_{z 1}}{4} \\
\frac{F_{z 2}}{4} & \frac{3 F_{z 2}}{4}
\end{array}\right] \text {, } \\
& G_{6}=\left[\begin{array}{cc}
k_{c}\left(k_{p}+k_{d} s\right) & 0 \\
0 & k_{c}\left(k_{p}+k_{d} s\right)
\end{array}\right] \text {, } \\
& G_{4}=\left[\begin{array}{cc}
\frac{3 k_{e} I_{10} s}{2 \delta_{0}\left(\left(R+k_{c}\right) \delta_{0}+2 k_{e} s\right)} & \frac{k_{e} I_{10} s}{2 \delta_{0}\left(\left(R+k_{c}\right) \delta_{0}+2 k_{e} s\right)} \\
\frac{k_{e} I_{20} s}{2 \delta_{0}\left(\left(R+k_{c}\right) \delta_{0}+2 k_{e} s\right)} & \frac{3 k_{e} I_{20} s}{2 \delta_{0}\left(\left(R+k_{c}\right) \delta_{0}+2 k_{e} s\right)}
\end{array}\right], \\
& G_{5}=\left[\begin{array}{cc}
\frac{\delta_{0}}{\left(R+k_{c}\right) \delta_{0}+2 k_{e} s} & 0 \\
0 & \frac{\delta_{0}}{\left(R+k_{c}\right) \delta_{0}+2 k_{e} s}
\end{array}\right] \text {. }
\end{aligned}
$$

\section{Conflict of Interests}

The authors declare that there is no conflict of interests regarding the publication of this paper.

\section{Acknowledgments}

This work is supported by the Natural Science Foundation of China (nos. 11202230 and 11302252) and National Key Technology R\&D Program of China (no. 2012BAG07B01-13).

\section{References}

[1] L. G. Yan, "Development and application of the Maglev transportation system," IEEE Transactions on Applied Superconductivity, vol. 18, no. 2, pp. 92-99, 2008. 
[2] D. M. Rote and Y. Cai, "Review of dynamic stability of repulsive-force maglev suspension systems," IEEE Transactions on Magnetics, vol. 38, no. 2, pp. 1383-1390, 2002.

[3] D. F. Zhou, C. H. Hansen, J. Li, and W. S. Chang, "Review of coupled vibration problems in EMS maglev vehicles," International Journal of Acoustics and Vibrations, vol. 15, no. 1, pp. 10-23, 2010.

[4] L. Yan, "Progress of the maglev transportation in China," IEEE Transactions on Applied Superconductivity, vol. 16, no. 2, pp. 1138-1141, 2006.

[5] M. Morita, M. Iwaya, and M. Fujino, "The characteristics of the levitation system of linimo (HSST system)," in Proceeding of the 18th International Conference on Magnetically Levitated System and Linear Drives, pp. 525-532, 2004.

[6] H.-S. Han, "A study on the dynamic modeling of a magnetic levitation vehicle," JSME International Journal, Series C: Mechanical Systems, Machine Elements and Manufacturing, vol. 46, no. 4, pp. 1497-1501, 2003.

[7] T. E. Alberts, G. Oleszczuk, and A. M. Hanasoge, "Stable levitation control of magnetically suspended vehicles with structural flexibility," in Proceedings of the American Control Conference (ACC '08), pp. 4035-4040, Seattle, Wash, USA, June 2008.

[8] R. M. Goodall, "Dynamics and control requirements for EMS maglev suspensions," in Proceeding of the 18th International Conference on Magnetically Levitated System and Linear Drives, pp. 925-934, Shanghai, China, 2004.

[9] A. E. Hajjaji and M. Ouladsine, "Modeling and nonlinear control of magnetic levitation systems," IEEE Transactions on Industrial Electronics, vol. 48, no. 4, pp. 831-838, 2001.

[10] C. M. Huang, J. Y. Yen, and M. S. Chen, "Adaptive nonlinear control of repulsive maglev suspension systems," Control Engineering Practice, vol. 8, no. 12, pp. 1357-1367, 2000.

[11] N. F. Al-Muthairi and M. Zribi, "Sliding mode control of a magnetic levitation system," Mathematical Problems in Engineering, vol. 2004, no. 2, pp. 93-107, 2004.

[12] L. Gentili and L. Marconi, "Robust nonlinear disturbance suppression of a magnetic levitation system," Automatica, vol. 39, no. 4, pp. 735-742, 2003.

[13] H. H. Rosenbrock, "Design of multivariable control systems using the inverse nyquist array," IEE Proceeding of Control Theory and Application, vol. 116, no. 11, pp. 1929-1936, 1969.

[14] Q.-G. Wang, Y. Zhang, and M.-S. Chiu, "Decoupling internal model control for multivariable systems with multiple time delays," Chemical Engineering Science, vol. 57, no. 1, pp. 115-124, 2002.

[15] H.-P. Huang and F.-Y. Lin, "Decoupling multivariable control with two degrees of freedom," Industrial and Engineering Chemistry Research, vol. 45, no. 9, pp. 3161-3173, 2006.

[16] J. Descusse and C. H. Moog, "Dynamic decoupling for rightinvertible nonlinear systems," Systems \& Control Letters, vol. 8, no. 4, pp. 345-349, 1987.

[17] G. Liu, P. Liu, Y. Shen, F. Wang, and M. Kang, "Experimental research on decoupling control of multi-motor variable frequency system based on neural network generalized inverse," in Proceedings of the IEEE International Conference on Networking, Sensing and Control (ICNSC '08), pp. 1476-1479, April 2008.

[18] B. T. Jevtović and M. R. Matauek, "PID controller design of TITO system based on ideal decoupler," Journal of Process Control, vol. 20, no. 7, pp. 869-876, 2010.

[19] P. Nordfeldt and T. Hägglund, "Decoupler and PID controller design of TITO systems," Journal of Process Control, vol. 16, no. 9, pp. 923-936, 2006.
[20] C. Rajapandiyan and M. Chidambaram, "Controller design for MIMO processes based on simple decoupled equivalent transfer functions and simplified decoupler," Industrial and Engineering Chemistry Research, vol. 51, no. 38, pp. 12398-12410, 2012.

[21] W.-J. Cai, W. Ni, M.-J. He, and C.-Y. Ni, "Normalized decoupling - a new approach for MIMO process control system design," Industrial and Engineering Chemistry Research, vol. 47, no. 19, pp. 7347-7356, 2008.

[22] Y. Shen, W. J. Cai, and S. Li, "Normalized decoupling control for high-dimensional MIMO processes for application in room temperature control HVAC systems," Control Engineering Practice, vol. 18, no. 6, pp. 652-664, 2010.

[23] Y. L. Shen, Y. X. Sun, and S. Y. Li, "Adjoint transfer matrix based decoupling control for multivariable processes," Industrial \& Engineering Chemistry Research, vol. 51, pp. 16419-16426, 2012.

[24] Q. Xiong, W.-J. Cai, and M.-J. He, "Equivalent transfer function method for PI/PID controller design of MIMO processes," Journal of Process Control, vol. 17, no. 8, pp. 665-673, 2007.

[25] F. Morilla, F. Vázquez, and J. Garrido, "Centralized PID control by decoupling for TITO processes," in Proceedings of the 13th IEEE International Conference on Emerging Technologies and Factory Automation (ETFA '08), pp. 1318-1325, 2008.

[26] G. He, J. Li, Y. Li, and P. Cui, "Interactions analysis in the maglev bogie with decentralized controllers using an effective relative gain array measure," in Proceedings of the 10th IEEE International Conference on Control and Automation (ICCA '13), pp. 1070-1075, Hangzhou, China, June 2013.

[27] D. F. Zhou, C. H. Hansen, and J. Li, "Suppression of maglev vehiclegirder self-excited vibration using a virtual tuned mass damper," Journal of Sound and Vibration, vol. 330, no. 5, pp. 883901, 2011.

[28] H. Guzmán, "Current loops in a magnetic levitation system," International Journal of Innovative Computing, Information and Control, vol. 5, no. 5, pp. 1275-1283, 2009.

[29] Y. Liu and W. Zhang, "Analytical design of two degree-offreedom decoupling control scheme for two-by-two systems with integrators," IET Control Theory and Applications, vol. 1, no. 5, pp. 1380-1389, 2007.

[30] Q.-G. Wang, Y. Zhang, and M.-S. Chiu, "Non-interacting control design for multivariable industrial processes," Journal of Process Control, vol. 13, no. 3, pp. 253-265, 2003.

[31] B. Kristiansson and B. Lennartson, "Evaluation and simple tuning of PID controllers with high-frequency robustness," Journal of Process Control, vol. 16, no. 2, pp. 91-102, 2006.

[32] R. Pintelon, P. Guillaume, Y. Rolain, J. Schoukens, and H. van Hamme, "Parametric identification of transfer functions in the frequency domain-a survey," IEEE Transactions on Automatic Control, vol. 39, no. 11, pp. 2245-2260, 1994.

[33] J. Garrido, F. Vázquez, and F. Morilla, "Centralized multivariable control by simplified decoupling," Journal of Process Control, vol. 22, no. 6, pp. 1044-1062, 2012.

[34] S. Skogestad and I. Postlethwaite, Multivariable Feedback Control: Analysis and Design, John Wiley \& Sons, New York, NY, USA, 2005. 


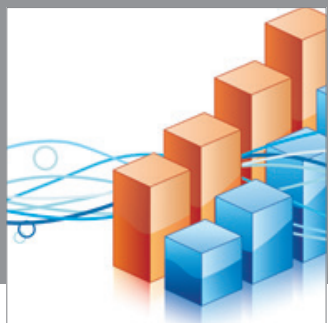

Advances in

Operations Research

mansans

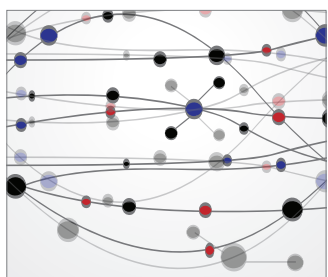

The Scientific World Journal
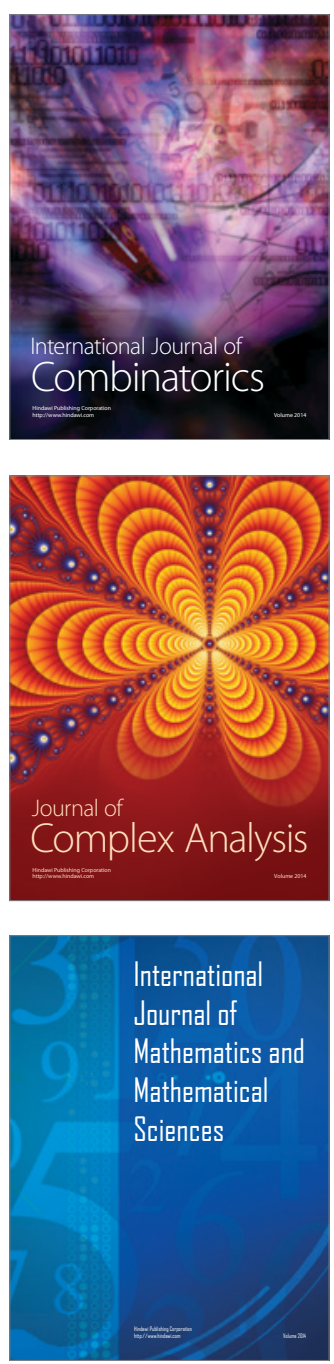
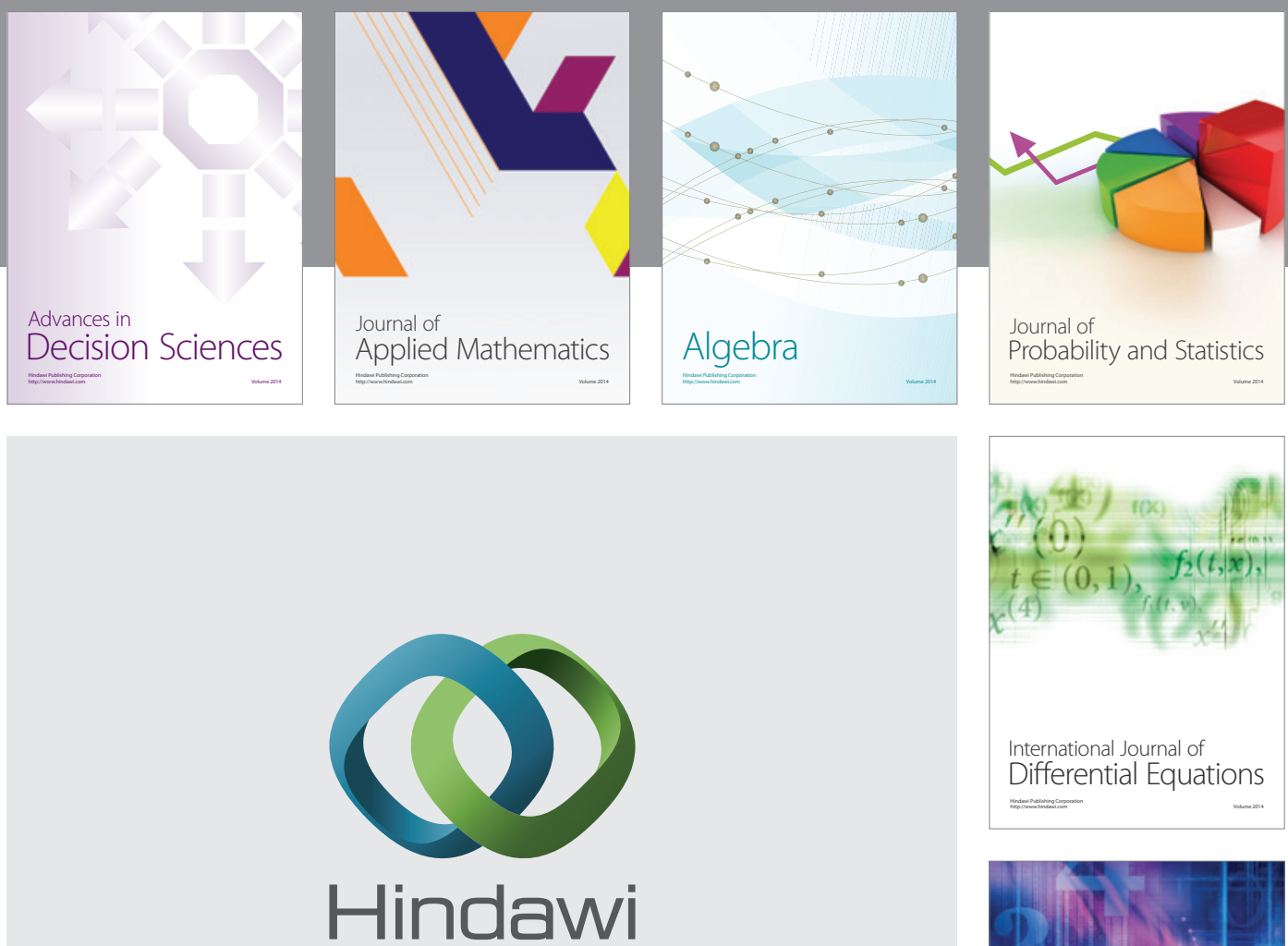

Submit your manuscripts at http://www.hindawi.com
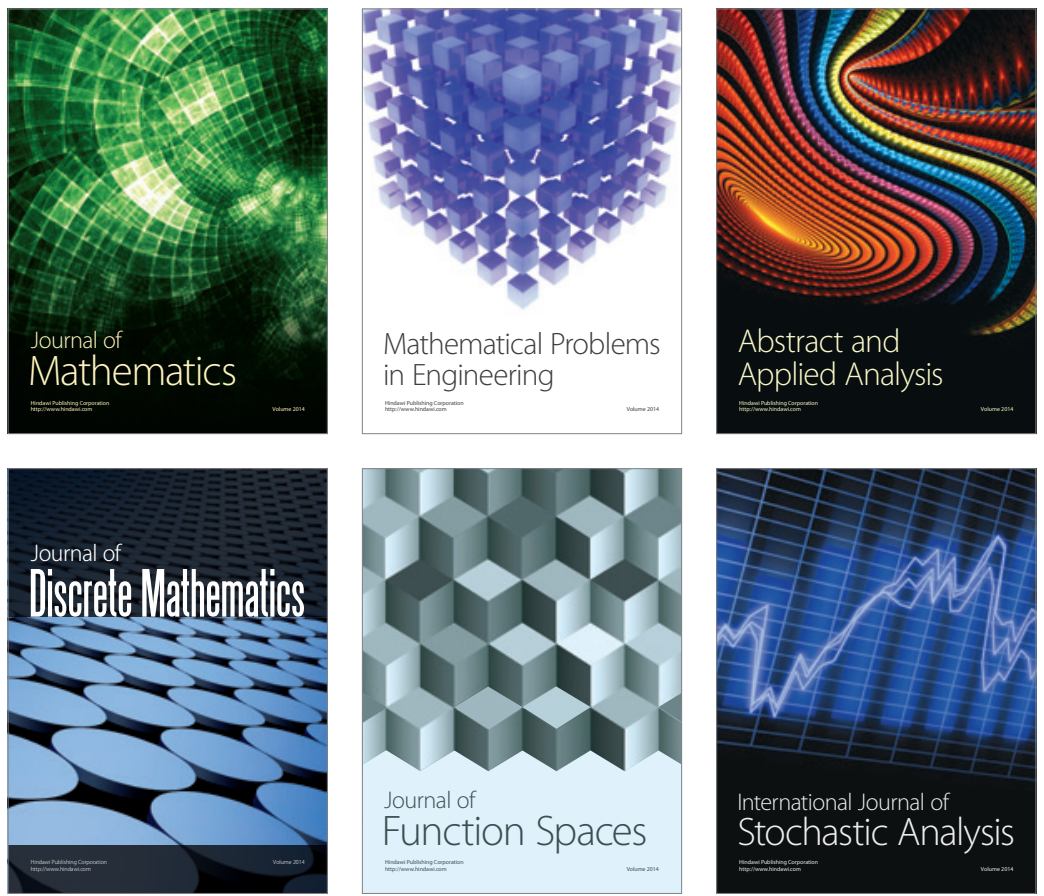

Journal of

Function Spaces

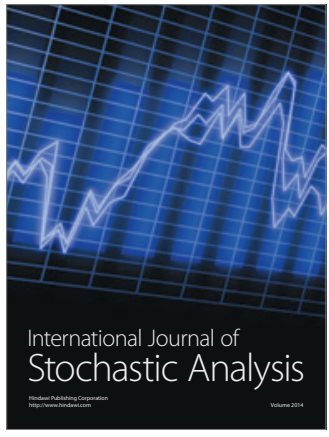

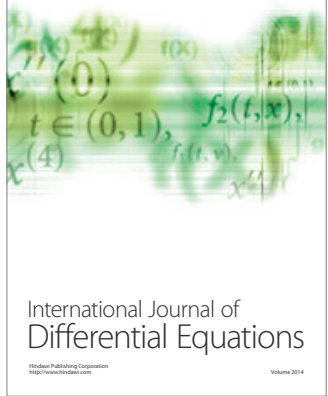
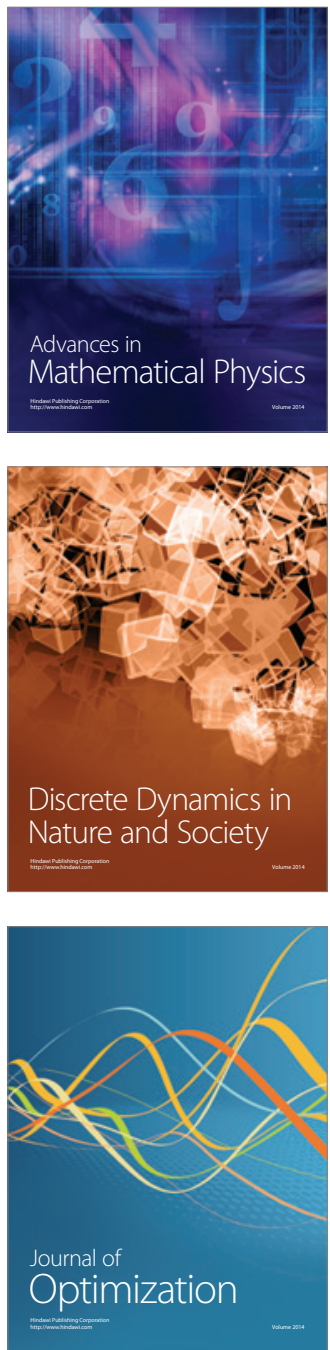